\title{
Crowned Dens Syndrome: A Case Report and Review of the Literature
}

\author{
Gwang Soo Lee, Ra Sun Kim, Hyung Ki Park, Jae Chil Chang \\ Department of Neurosurgery, Soonchunhyang University College of Medicine, Seoul, Korea
}

The crowned dens syndrome (CDS), also known as periodontoid calcium pyrophosphate dehydrate crystal deposition disease, is typified clinically by severe cervical pain, neck stiffness and atlantoaxial synovial calcification which could be misdiagnosed as meningitis, epidural abscess, polymyalgia rheumatica, giant cell arthritis, rheumatoid arthritis, cervical spondylitis or metastatic spinal tumor. Crystalline deposition on cervical vertebrae is less well known disease entity and only a limited number of cases have been reported to date. Authors report a case of CDS and describe the clinical feature.

Key Words: Neck pain - Calcium pyrophosphate $\cdot$ Axis $\cdot$ Computed tomography

\section{INTRODUCTION}

Firstly, the crowned dens syndrome (CDS) was named by Bouvet et al. in 1985 as it was presented by cervical pain due to deposition of calcium pyrophosphate dehydrate (CPPD) crystals or hydroxylapatite at cervical spine and shown radiologically as crown or halo-like distribution when radio-opaque density appeared at the top and side of the odontoid process ${ }^{4}$. CPPD crystal or basic calcium phosphate crystal is originated from hyaline cartilage which contains hypertropic chondrocyte, and it can arise from any joint that is composed of cartilage but rarely from tendon or bursa. If it occurs at cervicoaxial joint surrounded by several ligaments and if it causes severe pain and limitation of neck rotation at the same time, so it can be named as $\mathrm{CDS}^{3,13)}$. Authors report a case of CDS and review of the literature of this underrecognized condition.

\section{CASE REPORT}

A 49-year-old female visited the outpatient clinic with a 7-day history of acute onset severe posterior neck pain. On the day of symptom onset, she took an acupuncture and chiropractic manipulation of cervical spine which did not turn out to be helpful, and she experienced gradual worsening of pain

\footnotetext{
- Received: February 20, 2014 - Revised: March 4, 2014

- Accepted: March 4, 2014

Corresponding Author: Ra Sun Kim, MD

Department of Neurosurgery, Soonchunhyang University Hospital,

59 Daesagwan-ro, Yongsan-gu, Seoul 140-743, Korea

Tel: +82-2-709-9268, Fax: +82-2-792-5976

E-mail: rasonie@schmc.ac.kr

*This work was supported by the Soonchunhyang University Research Fund
}

and marked restriction of neck motion. There was no history of trauma and medical illness. On physical examination, passive cervical spine movements were significantly reduced without any focal neurologic deficit. Range of neck motion was limited to 20 degrees on rotation whereas extension and flexion were slightly diminished. At the end of the range of neck motion, pain was markedly provoked. Patient's vital signs were stable.

Plain radiographs of the cervical spine with open mouth view revealed abnormal radio-opaque lesion on the right side of the odontoid process (Fig. 1A). There was not any evidence of spinal instability on dynamic images. Computed tomography (CT) images showed atlantoaxial synovial calcifications in a crown or halo-like distribution around the odontoid process (Fig. 2A). Magnetic resonance image (MRI) showed low signal intensity around the odontoid process lesion on $\mathrm{T} 1$ weighted image but not involving itself (Fig. 3). Bone scan demonstrated increased radionuclide uptake around the odontoid process. We could not find any other lesion causative of pain within the spinal cord, nerve roots, intervertebral discs, cerebrospinal fluid and muscles. Laboratory tests revealed ele-
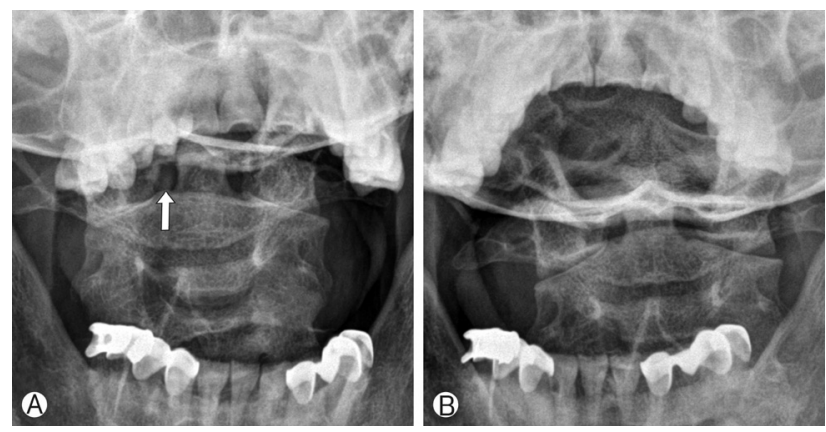

Fig. 1. Open mouth view demonstrates radio-opaque lesion beside the odontoid process (arrow) (A). Open mouth view checked in one month reveals pre-existing radio-opaque lesion disappeared (B). 


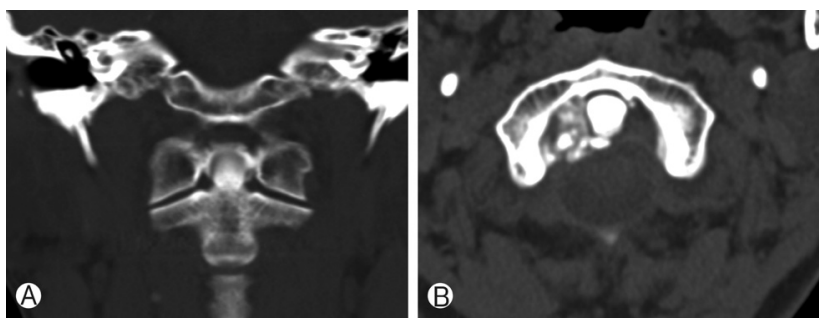

Fig. 2. Coronal $\mathrm{CT}$ scan at $\mathrm{Cl} / 2$ level demonstrates atlantoaxial synovial calcifications in a crown- or halo-like distribution around the odontoid process (A). Axial CT scan shows calcified lesion on the right side of periodontoid space and subchondral erosion (B).

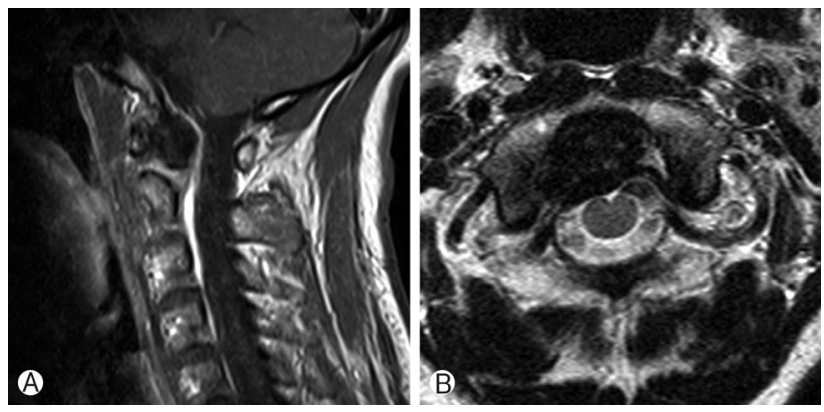

Fig. 3. Sagittal (A) and axial (B) T1 enhanced magnetic resonance image reveal non-enhancing, non-invasive mass like lesion around the odontoid process.

vated high sensitive C-reactive protein (hsCRP) of $1.97 \mathrm{mg} / \mathrm{dL}$ (normal range: $0.01-0.29 \mathrm{mg} / \mathrm{dL}$ ) while others were unremarkable. By integrating the result of tests listed above, we presumed a diagnosis as crowned dens syndrome.

Combination of prednisolone and nonsteroidal anti-inflammatory drug (NSAID) was taken and tapered off within three weeks, which led to resolve symptoms and normalize hsCRP. Neckbrace was put on to relieve motion-provoked pain during hospitalization. The lesion disappeared on plain radiographs followed in one month, which gave us a diagnostic clue to exclude such a condition from degenerative arthritis of the region (Fig. 1B).

\section{DISCUSSION}

The crowned dens syndrome is a rare disease entity which radiologically shows calcification at cruciform ligament around odontoid process and clinically presents acute cervico-occipital pain, fever, and neck stiffness accompanied by biological inflammatory reaction as mentioned earlier. In our case, clinical manifestations include severe posterior neck pain of acute onset, significant restriction of neck rotation and elevation of serum CRP. So that clinicians could make a confusion with spondyloarthritis around atlantoaxial joint area.
The duration of pain varies from few days to several weeks, the location can be from suboccipital area to inferior posterior neck, and the characteristics of pain are also diverse from mild neck discomfort to sleep-breaking severe pain ${ }^{5)}$. Most of the crystalline deposits are calcium pyrophosphate dehydrate (CPPD) crystals or hydroxyapatite crystal. There is no specific symptom when the crystal's size is small, but as it grows, it causes chronic cervical pain or spinal cord compression. Calcification can occur at not only cruciform ligament but also transverse, alar and apical ligament. Especially when CPPD crystal deposition occurs at ligamentum flavum, compressive cervical myelopathy can be presented ${ }^{8,16}$. Dirheimer et al. performed plain radiographs to 27 patients who had articular chondrocalcinosis but neurologically asymptomatic, and demonstrated that syndesmo-odontoid joint calcification was found in 12 patients $(44 \%)^{6}$. Constantin et al. performed CT scan to 21 patients with articular chodrocalcinosis, and found that 14 patients had calcifications at transverse ligament and 5 patients among the 14 presented neck pain ${ }^{5)}$.

CDS can be misdiagnosed as meningitis, epidural abscess, rheumatoid arthritis, polymyalgia rheumatica (PMR), giant cell arthritis (GCA), cervical spondylitis or metastatic spinal tumor ${ }^{1,15,18)}$. Especially, A. Aouba et al. emphasized that association with cervical stiffness and shoulder girdle pain or jaw claudication can be mislead to PMR or GCA ${ }^{1)}$, and Laborie $\mathrm{Y}$ et al. reported that chondrocalcinosis such as CDS can be included in the category of rheumatic disease from a widespectrum perspective. However, it should be noted that the pathophysiologic process has not been completely proven ${ }^{9}$, and therefore, caution should be used in relying on such statements or findings. There are several pathophysiologic theories about CPPD deposition at atlantoaxial joint. First, Constantin et al. set forth that chondroid cell in fibrous cartilage structures like meniscus of knee can be found in transverse ligament around odontoid process, and the presentation of these cell causes the disease ${ }^{5}$. Another theory is that fibroblast in cervical spine ligament transforms into chondrocyte and the transformation may cause calcification ${ }^{7}$.

For diagnosis, CT scan focused on C1-2 region is gold standard to detect calcification in transverse, apical and alar ligament. CT is even more helpful for small sized lesion when three-dimensional reconstruction is possible ${ }^{1,15,17,18)}$. CT scan seems to be superior to other diagnostic modality for ruling out CDS-like odontoid process fracture or Os odontoideum, and so forth. However, when CT scan is performed long time after the onset of symptom, calcification around odontoid process cannot be detected as it has been absorbed. So, CT scan should be done as soon as the symptom occurs ${ }^{10,20)}$. The presence of calcification can be assumed through plain radiograph by radio-opacity difference around odontoid process, 
but there is limitation as anatomical structures around odontoid process cannot be clearly distinguished. MRI is also helpful in identifying compressed spinal cord or soft tissues like nerve root, tendon, etc. What is interesting is that performing plain radiograph systemically over other joints like knee or wrist even though there is no specific symptom might be helpful because there were many cases which involve joints other than atlantoaxial joint in the CDS patients group ${ }^{19)}$. We couldn't find any abnormal joint except for periodontoid region. Elevation of ESR and CRP due to inflammatory reaction is common, and our case also showed slight elevation of CRP as $1.97 \mathrm{mg} / \mathrm{dL}$ which could be explained by time interval between symptom onset and blood sampling ${ }^{11}$.

Surgical procedure is performed rarely to patients who are diagnosed with CDS, but Baysal et al. demonstrates that 1 among 17 cases of CDS patients who progressively presented neurologic symptom and was treated by decompression surgery. And under microscopic examination, CPPD crystal in fibrous tissue was found and chronic inflammatory change was presented $^{2}$. In our case, when treated by combination therapy of prednisolone and NSAID, clinical symptoms showed dramatic improvement within 7 days. Although it was not clearly found that the period between the administration of NSAID and complete clinical recovery as many articles did not precisely describe, Ziza JM et al. and Resnick et al. reported that symptom had resolved on the $4^{\text {th }}$ day of administration and complete recovery had been made in 3 weeks ${ }^{14,20)}$. Reginato et al. reported that after the dramatic initial improvement within a week, there is a slow but persistent improvement in 3 to 5 weeks as the second phase, and it is the same at other lesions with microcrystalline joint. They also reported that colchicine seems to be effective, though not much as the effect of NSAID. There were debates about use of steroid, and low dose administration of steroid could be helpful to patients who have no effect from NSAID ${ }^{12}$.

\section{CONCLUSION}

Authors present a case of CDS as a rare cause of posterior neck pain with limitation of neck rotation. We suggest that clinicians should be aware of clinical feature of CDS for avoiding unnecessary lumbar puncture or biopsy, and cutting down hospitalization period.

\section{REFERENCES}

1. Aouba A, Vuillemin-Bodaghi V, Mutschler C, De Bandt M: Crowned dens syndrome misdiagnosed as polymyalgia rheumatica, giant cell arteritis, meningitis or spondylitis: an analysis of eight cases. Rheumatology (Oxford) 43:1508-1512, 2004
2. Baysal T, Baysal O, Kutlu R, Karaman I, Mizrak B: The crowned dens syndrome: a rare form of calcium pyrophosphate deposition disease. Eur Radiol 10:1003-1005, 2000

3. Beutler A, Rothfuss S, Clayburne G, Sieck M, Schumacher HR, Jr.: Calcium pyrophosphate dihydrate crystal deposition in synovium. Relationship to collagen fibers and chondrometaplasia. Arthritis Rheum 36:704-715, 1993

4. Bouvet JP, le Parc JM, Michalski B, Benlahrache C, Auquier $\mathrm{L}$ : Acute neck pain due to calcifications surrounding the odontoid process: the crowned dens syndrome. Arthritis Rheum 28: 1417-1420, 1985

5. Constantin A, Bouteiller G: Acute neck pain and fever as the first manifestation of chondrocalcinosis with calcification of the transverse ligament of the atlas. Five case-reports with a literature review. Rev Rhum Engl Ed 65:583-585, 1998

6. Dirheimer Y, Bensimon C, Christmann D, Wackenheim C: Syndesmo-odontoid joint and calcium pyrophosphate dihydrate deposition disease (CPPD). Neuroradiology 25:319-321, 1983

7. Doherty M, Dieppe P: Crystal deposition disease in the elderly. Clin Rheum Dis 12:97-116, 1986

8. Fye KH, Weinstein PR, Donald F: Compressive cervical myelopathy due to calcium pyrophosphate dihydrate deposition disease: report of a case and review of the literature. Arch Intern Med 159:189-193, 1999

9. Laborie Y, Berthelot JM: Rhizomelic pseudopolyarthritis: update. Rev Med Interne 23:518-532, 2002

10. Malca SA, Roche PH, Pellet W, Combalbert A: Crowned dens syndrome: a manifestation of hydroxy-apatite rheumatism. Acta Neurochir (Wien) 135:126-130, 1995

11. Ogata M, Ishikawa K, Ohira T: Cervical myelopathy in pseudogout. Case report. J Bone Joint Surg Am 66:1301-1303, 1984

12. Reginato AJ, Reginato AM: Disease associated with depositon of calcium pyrophosphate or hydroxyapatie, in Rubby S, Harris EDJ, Sledge CB (eds): Kelly's Textbook of Rheumatology, ed 6. Philadelphia: W.B.Saunders, Vol 1, pp 1377-1390, 2001

13. Reginato AJ, Schumacher HR, Martinez VA: The articular cartilage in familial chondrocalcinosis. Light and electron microscopic study. Arthritis Rheum 17:977-992, 1974

14. Resnick D, Pineda C: Vertebral involvement in calcium pyrophosphate dihydrate crystal deposition disease. Radiographicpathological correlation. Radiology 153:55-60, 1984

15. Salaffi F, Carotti M, Guglielmi G, Passarini G, Grassi W: The crowned dens syndrome as a cause of neck pain: clinical and computed tomography study in patients with calcium pyrophosphate dihydrate deposition disease. Clin Exp Rheumatol 26: 1040-1046, 2008

16. Sato R, Takahashi M, Yamashita Y, Izunaga H, Sakamoto Y, Yamamoto M, et al: Calcium crystal deposition in cervical ligamentum flavum: CT and MR findings. J Comput Assist Tomogr 16:352-355, 1992

17. Scutellari PN, Galeotti R, Leprotti S, Ridolfi M, Franciosi R, Antinolfi G: The crowned dens syndrome. Evaluation with CT imaging. Radiol Med 112:195-207, 2007

18. Wu DW, Reginato AJ, Torriani M, Robinson DR, Reginato AM: The crowned dens syndrome as a cause of neck pain: report of two new cases and review of the literature. Arthritis Rheum 53:133-137, 2005

19. Zapletal J, Hekster RE, Straver JS, Wilmink JT, Hermans J: Association of transverse ligament calcification with anterior atlanto-odontoid osteoarthritis: CT findings. Neuroradiology 37: 667-669, 1995

20. Ziza JM, Bouvet JP, Auquier L: Acute suboccipital neck pain of calcifying origin. Rev Rhum Mal Osteoartic 49:549-551, 1982 